\title{
Erratum to: Progress in Intelligent Computing Techniques: Theory, Practice, and Applications
}

Pankaj Kumar Sa, Manmath Narayan Sahoo, M. Murugappan, Yulei Wu and Banshidhar Majhi

Erratum to:

P.K. Sa et al. (eds.), Progress in Intelligent Computing Techniques: Theory, Practice, and Applications, Advances in Intelligent Systems and Computing 719, https://doi.org/10.1007/978-981-10-3376-6

The original version of the book was inadvertently published with incorrect volume number 519 which has been now corrected as 719 .

The updated online version of this book can be found at

https://doi.org/10.1007/978-981-10-3376-6 